\title{
Domain Wall Resistance based on Landauer's Formula
}

\author{
Gen TATARA \\ Graduate School of Science, Osaka University, Toyonaka, Osaka 560-0043
}

\begin{abstract}
The scattering of the electron by a domain wall in a nano-wire is calculated perturbatively to the lowest order. The resistance is calculated by use of Landauer's formula. The result is shown to agree with the result of the linear response theory if the equilibrium is assumed in the four-terminal case.
\end{abstract}

\section{INTRODUCTION}

The electronic transport properties in nano-size ferromagnetic structures has been drawing growing interest recently. Of most interest would be the magnetoresistance effect, namely, the change of the resistance by application of a magnetic field. The magnetoresistance in nano-structures is due to the rearrangement of the magnetization, namely the creation and motion of domain walls, driven by the external field. Recently high-sensitivity measurements on magnetic nano-structures such as wires and contacts has become possible and intensive experimental studies of the

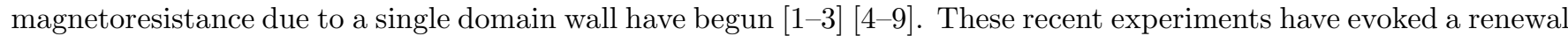
of interest in theories of the scattering of the electron by a domain wall [7, 10 15] [16, 17, although the first theoretical study of this problem goes back to more than 25 years ago [18].

The scattering mechanism is due to the non-adiabatic deviation of the electron spin from the local magnetization as the electron goes through the wall. In the case of a wall with thickness $\lambda \gg k_{F}^{-1}$ ( $k_{F}$ being the Fermi wave length), the electron spin can adiabatically follow the spatial rotation of the magnetization, resulting in a negligiblly small reflection. This is the reason the wall contributes very little in the magnetoresistance in the $3 d$ metals in the bulk [18].

The situation changes in nano-magnets, where the profile of the wall can be different from that in the bulk magnets. The extreme case would be an atomic size contact. There the wall is believed to be constrained in a small contact region of an atomic scale due to a rapid change of the contact shape [19], and thus the adiabaticity does not hold resulting in a strong scattering [14,7]. Indeed an experiment on $\mathrm{Ni}$ contact revealed a magnetoresistance of about $300 \%$ at room temperature [5], which is significantly large compared with other giant magnetoresistive materials such as multilayers. The result has been interpreted as due to a strong electron scattering by a domain wall, which is trapped in the narrow contact region in the absence of the magnetic field, and which is expelled by applying a magnetic field [7].

In this paper the domain wall resistance in nano-wires or contacts is calculated by use of Landauer's formula. The advantage of this formalism is that we can see how the density of states of the sample and the lead appears in the resistance, which is not possible in he linear response calculation as carried out in ref. (7). The system is treated as in one dimension and the calculation is at zero temperature. The transmission coefficients are calculated in section 2 , and the resistance is calculated in the case of four- and two-terminals in section 3 . The result is shown to agree with the result of the Mori formula of the linear response theory in the case of the equilibrium flow in a four-terminal case.

Throughout the paper we consider only channels which contribute to the transport and so do not discuss the quantized behavior of the conductance.

The phase of the transmission coefficient contains important information on the stability of the electron system. This has been considered elsewhere [20].

\section{SCATTERING PROPERTIES}

We consider the exchange interaction between the electron spin and the local spin. The Hamiltonian is

$$
H=\sum_{k \sigma} \epsilon_{k} c_{k \sigma}^{\dagger} c_{k \sigma}-g \int d x \mathbf{S}(x)\left(c^{\dagger} \boldsymbol{\sigma} c\right)
$$

where $\epsilon_{k} \equiv \hbar^{2} k^{2} / 2 m-\epsilon_{F}\left(\epsilon_{F}\right.$ being the Fermi energy). The spin index is denoted by $\sigma= \pm$ and $\sigma$ is the Pauli matrix (spin indices are suppressed in the second term). The local spin $\mathbf{S}$ has a spatial dependence of a domain wall. In terms of a polar coordinate $\left(S_{z} \equiv S \cos \theta\right)$ the wall is represented as $\cos \theta \rightarrow 1$ for $x \ll-\lambda$ and $\cos \theta \rightarrow-1$ for $x \gg \lambda, \lambda$ being the thickness of the wall. We consider two typical profiles of the wall; 


$$
\begin{aligned}
\text { bulk wall }: \cos \theta & =\tanh (x / \lambda) \\
\text { linear wall }: \cos \theta & =\left\{\begin{array}{cc}
1 & (x>\lambda) \\
x / \lambda & (-\lambda \leq x \leq \lambda), \\
-1 & (x<-\lambda)
\end{array}\right.
\end{aligned}
$$

where the first case corresponds to the wall determined by the uniaxial anisotropy energy, which would be the case of bulk magnets, and the second linear profile would be realized in small contacts.

To proceed we carry out a local gauge transformation in the electron spin space so that the $z$-axis is chosen to be along the local direction of spin $\vec{S}$ [21]. The transformation is written as $a_{\sigma}=(U c)_{\sigma}$, where $U$ is a position-dependent matrix defined as $U \equiv \cos \left(\frac{\theta}{2}\right) \sigma_{z}+\sin \left(\frac{\theta}{2}\right) \sigma_{y}$ and the electron in the new frame is denoted by $a_{\sigma}$. The Hamiltonian is transformed to be $H=H_{0}+H_{\text {int }}$ where $H_{0} \equiv \sum_{k \sigma} \epsilon_{k \sigma} a_{k \sigma}^{\dagger} a_{k \sigma}$ and $\epsilon_{k \sigma} \equiv \epsilon_{k}-\sigma \Delta$ is the energy of uniformly polarised electron with the exchange splitting $\Delta \equiv g|\mathbf{S}|$. The term $H_{\text {int }}$ describes the interaction between the electron and the wall and is given by [1]

$$
H_{\mathrm{int}} \equiv \frac{\hbar^{2}}{2 m} \frac{1}{L} \sum_{k q}\left[-\left(k+\frac{q}{2}\right) A_{q} a_{k+q}^{\dagger} \sigma_{x} a_{k}+\frac{1}{4 L} \sum_{p} A_{p} A_{-p+q} a_{k+q}^{\dagger} a_{k}\right],
$$

where

$$
A_{q} \equiv \int d x e^{-i q x} \nabla \theta
$$

is a domain-wall form factor. Note that $\nabla \theta \rightarrow 0$ for $|x| \rightarrow \infty$ and so the interaction is localized near the wall. The spin-conserving process in the rotated frame as the electron goes through the wall corresponds to the adiabatic process, where the electron spin follows the rotation of the magnetization, and the spin flip corresponds to the deviation from the adiabaticity.

The interaction $H_{\text {int }}$ leads to a scattering of the electron and thus contributes to a resistivity due to the wall as has been discussed before based on a linear response theory [11,15, 17. Here we take a different approach of calculating the $T$-matrix perturbatively with respect to $H_{\text {int }}$. The solution of the Schrödinger equation with the energy of $E$, which we denote by $\left|\Psi_{E}\right\rangle$ (two component vector corresponding to two spin state), is given as

$$
\left|\Psi_{E}>=\right| \Psi_{0}>+G_{E}^{0} T_{E} \mid \Psi_{0}>
$$

where $\mid \Psi_{0}>$ is the incoming wave, $G_{E}^{0} \equiv \frac{-1}{H_{0}-E-i 0}$ is the free Green function with energy $E$ and $T$-operator is defined as $T_{E} \equiv H_{\mathrm{int}} \frac{1}{1-G_{E}^{0} H_{\mathrm{int}}}$. The small imaginary part in $G_{E}^{0}$ is to choose the correct boundary condition of outgoing wave. We consider the incoming wave with energy $E$ and spin $\sigma$. They are explicitly written as

$$
<x\left|\Psi_{0}^{\uparrow}>=\left(\begin{array}{c}
e^{i k_{+} x} \\
0
\end{array}\right),<x\right| \Psi_{0}^{\downarrow}>=\left(\begin{array}{c}
0 \\
e^{i k_{-} x}
\end{array}\right),
$$

where $k_{\sigma} \equiv \sqrt{2 m(E+\sigma \Delta)}$ is the wave vector of the spin $\sigma$ component. The spin $\sigma^{\prime}$ component of the full wave function for the incident wave $\mid \Psi_{0}^{\sigma}>$ is then obtained from eq. (6) as

$$
\Psi_{E}^{\sigma^{\prime} \sigma}(x)=\delta_{\sigma^{\prime}, \sigma} e^{i k_{\sigma} x}+\int d x^{\prime} G_{E \sigma^{\prime}}^{0}\left(x-x^{\prime}\right) \sum_{k^{\prime}} e^{i k^{\prime} x^{\prime}} T_{k^{\prime} \sigma^{\prime}, k_{\sigma} \sigma},
$$

where $G_{E \sigma^{\prime}}^{0}\left(x-x^{\prime}\right) \equiv<x \sigma^{\prime}\left|G_{E}^{0}\right| x^{\prime} \sigma^{\prime}>=-i \frac{m}{\left|k_{\sigma^{\prime}}\right|} e^{i k_{\sigma^{\prime}}\left|x-x^{\prime}\right|}$ and $T_{k^{\prime} \sigma^{\prime}, k_{\sigma} \sigma} \equiv<k^{\prime} \sigma^{\prime}\left|T_{E}\right| k_{\sigma} \sigma>$.

The transmission and reflection coefficients are defined from the asymptotic behavior as

$$
\Psi_{E}^{\sigma^{\prime} \sigma}(x) \rightarrow\left\{\begin{array}{cc}
\delta_{\sigma^{\prime} \sigma} e^{i k_{\sigma} x}+r_{\sigma^{\prime} \sigma} e^{-i k_{\sigma^{\prime}} x} & (x \rightarrow-\infty) \\
t_{\sigma^{\prime} \sigma} e^{i k_{\sigma^{\prime}} x} & (x \rightarrow+\infty)
\end{array}\right.
$$

The result is

$$
\begin{aligned}
& t_{\sigma^{\prime} \sigma}\left(k_{\sigma}\right)=\delta_{\sigma^{\prime} \sigma}-i \frac{m L}{\left|k_{\sigma^{\prime}}\right|} T_{k_{\sigma^{\prime} \sigma^{\prime}, k_{\sigma} \sigma}} \\
& r_{\sigma^{\prime} \sigma}\left(k_{\sigma}\right)=-i \frac{m L}{\left|k_{\sigma^{\prime}}\right|} T_{-k_{\sigma^{\prime} \sigma^{\prime}, k_{\sigma} \sigma}} .
\end{aligned}
$$


The diagonal (in spin) component of the $T$-matrix for the incoming wave vector of $k_{\sigma}$ is calculated as

$$
\begin{aligned}
T_{k_{\sigma} \sigma, k_{\sigma} \sigma}= & <k_{\sigma}, \sigma\left|H_{\mathrm{int}}\right| k_{\sigma}, \sigma>+\sum_{k^{\prime}}<k_{\sigma}, \sigma\left|H_{\mathrm{int}}\right| k^{\prime},-\sigma><k^{\prime},-\sigma\left|G_{E}^{0}\right| k^{\prime},-\sigma><k^{\prime},-\sigma\left|H_{\mathrm{int}}\right| k_{\sigma}, \sigma> \\
& +O\left(A^{4}\right) \\
= & \frac{1}{8 m L^{2}} \sum_{q}\left|A_{q}\right|^{2}\left[1-\frac{\left(2 k_{\sigma}+q\right)^{2}}{\left(q+k_{\sigma}\right)^{2}-k_{-\sigma}^{2}-i 0}\right]+O\left(A^{4}\right) .
\end{aligned}
$$

Off-diagonal components are given by

$$
T_{ \pm k_{-\sigma},-\sigma, k_{\sigma} \sigma}=\frac{\left(k_{\sigma} \pm k_{-\sigma}\right)}{4 m L} A_{ \pm k_{-\sigma}-k_{\sigma}}+O\left(A^{3}\right) .
$$

The result of the transmission and reflection coefficients are

$$
\begin{aligned}
t_{\sigma \sigma}\left(k_{\sigma}\right) & =1+i \frac{1}{8 k_{\sigma} L} \sum_{q}\left|A_{q}\right|^{2} \frac{3 k_{\sigma}^{2}+k_{-\sigma}^{2}+2 k_{\sigma} q}{\left(q+k_{\sigma}\right)^{2}-k_{-\sigma}^{2}-i 0} \\
t_{-\sigma, \sigma}\left(k_{\sigma}\right) & =-i \frac{\left(k_{\sigma}+k_{-\sigma}\right)}{4 k_{-\sigma}} A_{-k_{\sigma}+k_{-\sigma}} \\
r_{-\sigma, \sigma}\left(k_{\sigma}\right) & =-i \frac{\left(k_{\sigma}-k_{-\sigma}\right)}{4 k_{-\sigma}} A_{-k_{\sigma}-k_{-\sigma}} \\
r_{\sigma, \sigma}\left(k_{\sigma}\right) & =O\left(A^{2}\right) .
\end{aligned}
$$

The scattering probabilities are defined as $T_{\sigma^{\prime} \sigma} \equiv\left|t_{\sigma^{\prime} \sigma}\right|^{2}$ and $R_{\sigma^{\prime} \sigma} \equiv\left|r_{\sigma^{\prime} \sigma}\right|^{2}$. These probabilities for the electron at Fermi level (i.e., $k_{\sigma}=k_{F \sigma}$ ) are obtained to the second order in $A$ as

$$
\begin{aligned}
T_{\sigma \sigma} & =1-\frac{1}{4\left(1-\zeta^{2}\right)}\left(\zeta^{2}\left|A_{+}\right|^{2}+\left|A_{-}\right|^{2}\right) \\
T_{-\sigma, \sigma} & =\frac{1}{4(1-\sigma \zeta)^{2}}\left|A_{-}\right|^{2} \\
R_{-\sigma, \sigma} & =\frac{1}{4(1-\sigma \zeta)^{2}}\left|A_{+}\right|^{2}
\end{aligned}
$$

and $R_{\sigma \sigma}=O\left(A^{4}\right)$, where $A_{+} \equiv A_{k_{F+}+k_{F-}}\left(=A_{2 k_{F}}\right)$ and $A_{-} \equiv A_{k_{F+}-k_{F-}}\left(=A_{2 k_{F} \zeta}\right)$ describes a backward and forward scattering with spin flip, respectively $\left(k_{F} \equiv\left(k_{F+}+k_{F-}\right) / 2\right.$ and $\left.\zeta \equiv\left(k_{F+}-k_{F-}\right) /\left(k_{F+}+k_{F-}\right)\right)$. The conservation of the current,

$$
k_{F \sigma}=k_{F \sigma} T_{\sigma \sigma}+k_{F,-\sigma} T_{-\sigma, \sigma}+k_{F,-\sigma} R_{-\sigma, \sigma},
$$

is satisfied.

\section{WALL RESISTANCE}

To relate these quantities to resistance, we consider a four-terminal case where the system is coupled with two leads, $L_{1}$ and $L_{2}$ and two terminals, $T_{A}$ and $T_{B}$ (Fig. 11). (The gauge transformation does not affect the resistance, since the shift of the current due to the transformation vanishes far away from the wall.) $L_{1}$ and $L_{2}$ are connected with a reserver (current source) and $T_{A}$ and $T_{B}$ are to measure the voltage difference across the sample. The argument goes similarly to the non-magnetic case described in refs. 22, 23 but taking account of spin dependence. The chemical potential of $L_{1}$ and $L_{2}$ are fixed to be $\mu_{1} \equiv \epsilon_{F}+e V_{0}$ and $\mu_{2} \equiv \epsilon_{F}$ (V $V_{0}$ is the applied voltage), respectively. The chemical potential of $T_{A}$ and $T_{B}, \mu_{A}$ and $\mu_{B}$ are determined below by the condition of vanishing current flow through each terminal. The true voltage difference across the sample is different from $V_{0}$ and is given as $V \equiv\left(\mu_{A}-\mu_{B}\right) / e$. The resistance of the system is written as $R_{\mathrm{w}} \equiv V / I, I$ being the current through the sample.

We first consider the case where the leads are ferromagnets with density of states for the spin state $\sigma$ given by $N_{\sigma}$. The case of non-magnetic leads is discussed at the end of this section. The number of the electron with spin $\sigma$ injected from $L_{1}$ is $N_{\sigma} e V_{0}$, and thus the electric current is written by use of transmission probability as 


$$
I=e V \sum_{\sigma, \sigma^{\prime}} N_{\sigma} \frac{e \hbar}{m L} k_{F \sigma^{\prime}} T_{\sigma^{\prime} \sigma}
$$

The voltage $\mu_{A}$ and $\mu_{B}$ are determined by the balance between the terminal and lead. In $L_{1}$, the density (per energy) of spin $\sigma$ electron is written as

$$
n_{1 \sigma}=\frac{1}{2}\left(N_{\sigma}\left(1+R_{\sigma \sigma}\right)+N_{-\sigma} R_{\sigma,-\sigma}\right)
$$

Here a factor of $1 / 2$ comes from choosing right moving electron. The density at the other lead is given by

$$
n_{2 \sigma}=\frac{1}{2}\left(N_{\sigma} T_{\sigma \sigma}+N_{-\sigma} T_{\sigma,-\sigma}\right)
$$

The condition of vanishing flow between $L_{1}$ and $T_{A}$ and $L_{2}$ and $T_{B}$ are given as

$$
\begin{aligned}
\sum_{\sigma} n_{1 \sigma}\left(\mu_{1}-\mu_{A}\right) & =\sum_{\sigma}\left(N_{\sigma}-n_{1 \sigma}\right)\left(\mu_{A}-\mu_{2}\right) \\
\sum_{\sigma} n_{2 \sigma}\left(\mu_{1}-\mu_{B}\right) & =\sum_{\sigma}\left(N_{\sigma}-n_{2 \sigma}\right)\left(\mu_{B}-\mu_{2}\right),
\end{aligned}
$$

respectively. This leads to the voltage difference across the sample of

$$
\begin{aligned}
V & =\frac{1}{e} \sum_{\sigma}\left(n_{1 \sigma}-n_{2 \sigma}\right)\left(\mu_{1}-\mu_{2}\right) \\
& =\frac{V}{2} \frac{1}{N_{+}+N_{-}} \sum_{\sigma} N_{\sigma}\left(1+R_{\sigma \sigma}-T_{\sigma \sigma}+R_{-\sigma, \sigma}-T_{-\sigma, \sigma}\right) .
\end{aligned}
$$

By use of eq. (14) the resistance to the lowest order in domain wall scattering is obtained as

$$
R_{\mathrm{w}}=\frac{m L}{2 e^{2} \hbar} \frac{1}{\sum_{\sigma} N_{\sigma} k_{F \sigma}} \frac{1}{\left(1-\zeta^{2}\right)^{2}}\left[\zeta^{2}(1+\zeta \delta)\left|A_{+}\right|^{2}-\left(\zeta^{2}+\zeta \delta\right)\left|A_{-}\right|^{2}\right],
$$

where $\delta \equiv\left(N_{+}-N_{-}\right) /\left(N_{+}+N_{-}\right)$. Naively it appears correct to choose $N_{\sigma}=\frac{L m}{\pi \hbar^{2} k_{F \sigma}} \equiv D_{\sigma}$, equal to the onedimensional density of state. This is wrong if the equilibrium situation with a steady current flow is considered. In fact, the electron with down spin, which has a smaller velocity of $k_{F-}$, cannot leave the scattering region quickly after the scattering and thus the density becomes higher by a factor proportional to $1 / k_{F-}$ (see Fig. 2). Therefore under a steady flow, the effective density of states becomes proportional to $D_{\sigma} / k_{F \sigma}$, namely, $N_{\sigma}=D_{\sigma} \frac{\left(1-\zeta^{2}\right)}{\left(1+\zeta^{2}\right)} \frac{1}{(1+\sigma \zeta)}$, where a prefactor is chosen to conserve the total density, i.e., $N_{+}+N_{-}=D_{+}+D_{-}$. This point has not been discussed in the multi-channel case carried out so far [22]. Then $\delta=-\frac{2 \zeta}{1+\zeta^{2}}$ and we obtain

$$
R_{\mathrm{w}}=\frac{h}{e^{2}} \frac{\zeta^{2}}{8\left(1-\zeta^{2}\right)}\left[\left|A_{+}\right|^{2}+\left|A_{-}\right|^{2}\right] .
$$

This result is the same as that obtained based on a linear response theory [7], where the equilibrium is assumed.

In the case of two-terminals, the system is out of equilibrium and the resistance is modified. The conductance in this case is given as the transmitted current, eq. (16) with $N_{\sigma}=D_{\sigma}$, divided by the applied voltage, $V$. Thus

$$
G=\frac{e^{2}}{h} \sum_{\sigma}\left(T_{\sigma \sigma}+\frac{k_{F,-\sigma}}{k_{F \sigma}} T_{-\sigma, \sigma}\right) .
$$

The wall resistance is written as $R_{\mathrm{w}}^{(2 \mathrm{t})} \equiv G^{-1}-G_{0}^{-1}$, where $G_{0} \equiv \frac{2 e^{2}}{h}$ is the conductance without the wall. Thus to the lowest order in the wall scattering,

$$
R_{\mathrm{w}}^{(2 \mathrm{t})}=\frac{h}{e^{2}} \frac{\zeta^{2}}{4\left(1-\zeta^{2}\right)}\left|A_{+}\right|^{2}
$$

This resistance contains only backward scattering $k_{F \sigma} \rightarrow-k_{F,-\sigma}$. This result is obtained from the four terminal case, eq. (21), by putting $N_{\sigma}=D_{\sigma}$. 
Two resistances, (22) and (24) (dashed and solid lines, respectively), are plotted in Fig. 3 for the two profiles, (2) and (3). In the bulk case, (2), $A_{q}$ is given by

$$
A_{q}=\frac{\pi}{\cosh (\pi q \lambda / 2)}
$$

and so resistance decays rapidly for $|q| \gtrsim \lambda^{-1}$. In the case of a linear wall, (3), decay is slower (Fig. (3);

$$
A_{q}=\pi \frac{\sin q \lambda}{q \lambda}
$$

This difference in the form factor leads to a slightly different resistance, but strong suppression of the resistance for $k_{F} \lambda \gg 1$ is universal. The difference between the two- and four-terminal cases is small if the Zeeman splitting is large but becomes manifest when $\zeta$ is small.

Let us mention the case where the leads connected to the sample are non-magnetic metals. If we write the hopping probability between the lead and the sample as $T_{\sigma}^{(\mathrm{L})}$, the balance of the density of states requires $D T_{\sigma}^{(\mathrm{L})}=N_{\sigma}$, where $D$ is the density of states in the lead. Thus the voltage across the sample is determined by the same eqs. as (17)-(20), and so the resistance is equal to that for the ferromagnetic lead discussed above.

\section{SUMMARY}

The scattering of the electron by a domain wall is studied theoretically. By use of a local gauge transformation, the interaction between the electron and the wall is treated perturbatively to the lowest order. The transmission coefficients are obtained and the resistance was calculated using the Laundauer's formula with four-terminals. The comparison with the result of linear response theory was made.

\section{ACKNOWLEDGMENTS}

The author thanks H. Matsukawa and Y. Tokura for stimulating discussion. He also thanks Max Planck Institut fur Mikrostrukturphysik for its hospitality during his stay at the early stage of this work and Alexander von Humboldt Stiftung for financial support. This work is supported by a Grand-in-Aid for Scientific Research from the Ministry of Education, Science, Sports and Culture.

[1] K. Hong and N. Giordano: Euro. Phys. Lett. 36 (1996) 147.

[2] Y. Otani, K. Fukamichi, O. Kitakami, Y. Shimada, B. Pannetier, J. P. Nozieres, T. Matsuda and A. Tonomura: Proc. MRS Spring Meeting, San Francisco, 1997.

[3] U. Ruediger, J. Y. S. Zhang, A. D. Kent and S. S. P. Parkin: Phys. Rev. Lett. 80 (1998) 5639.

[4] U. Ruediger, J. Yu, L. Thomas, S. S. P. Parkin, A. D. Kent: Phys. Rev. B59 (1999) 11914.

[5] N. Garcia, M. Munoz and Y. W. Zhao: Phys. Rev. Lett. 82 (1999) 2923.

[6] T. Ono, Y. Ooka, H. Miyajima and Y. Otani: Appl. Phys. Lett. 75 (1999) 1622.

[7] G. Tatara, Y.-W. Zhao, M. Muñoz and N. García: Phys. Rev. Lett. 83 (1999) 2030.

[8] F. Komori and K. Nakatsuji: J. Phys. Soc. Jpn. 68 (1999) 3786.

[9] Y. W. Zhao, M. Munoz, G. Tatara and N. Garcia: preprint (1999).

[10] M. Yamanaka and N. Nagaosa: J. Phys. Soc. Jpn. 65 (1996) 3088.

[11] G. Tatara and H. Fukuyama: Phys. Rev. Lett. 78 (1997) 3773.

[12] P. M. Levy and S. Zhang, Phys. Rev. Lett. 79 (1997) 5110.

[13] Y. L.-Geller, I. L. Aleiner and P. M. Goldbart: Phys. Rev. Lett. 81 (1998) 3215.

[14] J. B. A. N. van Hoof, K. M. Schep, A. Brataas, G. Bauer and P. J. Kelly: Phys. Rev. B59 (1999) 138.

[15] A. Brataas, G. Tatara and G. Bauer: Phys. Rev. B60 (1999) 3406.

[16] H. Imamura, N. Kobayashi, S. Takahashi and S. Maekawa: Phys. Rev. Lett. 84 (2000) 1003.

[17] K. Nakanishi and Y. Nakamura: Phys. Rev. B61 (2000) 11278.

[18] G. G. Cabrera and L. M. Falicov: Phys. Stat. Sol. (b)61 (1974) 539. 
[19] P. Bruno: Phys. Rev. Lett. 83 (1999) 2425.

[20] G. Tatara and Y. Tokura, preprint.

[21] G. Tatara and H. Fukuyama: Phys. Rev. Lett. 72 (1994) 772; J. Phys. Soc. Jpn. 63 (1994) 2538.

[22] M. Buttiker, Y. Imry, . Landauer and S. Pinhas: Phys. Rev. B31 (1985) 6207.

[23] Y. Imry, "Introduction to mesoscopic physics", Oxford Univ. Press (1997) Chapter 5.

FIG. 1. The system coupled with four terminals. $L_{1}$ and $L_{2}$ are leads with chemical potential $\mu_{1} \equiv \epsilon_{F}+e V_{0}$ and $\mu_{2} \equiv \epsilon_{F}$, respectively, which are coupled with an infinitely large current source. $T_{A}$ and $T_{B}$ are the terminals to measure voltage whose chemical potential is denoted by $\mu_{A}$ and $\mu_{B}$, respectively. The voltage of the sample is given by $V=\left(\mu_{A}-\mu_{B}\right) / e$.

FIG. 2. Schematic picture of an equilibrium flow in the scattering of particles with two components with different velocities. If some portion of the incoming particle with velocity $\hbar k_{F+} / m$ is scattered to have a smaller velocity of $\hbar k_{F-} / m$, the density of these outgoing particle becomes higher.

FIG. 3. Resistances by the wall for two different profiles of the wall (bulk wall given by eq. (2) (upper graph) and linear wall, (3) (lower graph)) plotted as functions of $\lambda$ for $\zeta=0.3$ and 0.8 . Solid and dashed lines corresponds to the four-terminal case $\left(R_{\mathrm{w}}\right)$ and two-terminal case $\left(R_{\mathrm{w}}^{(2 \mathrm{t})}\right)$, respectively. 


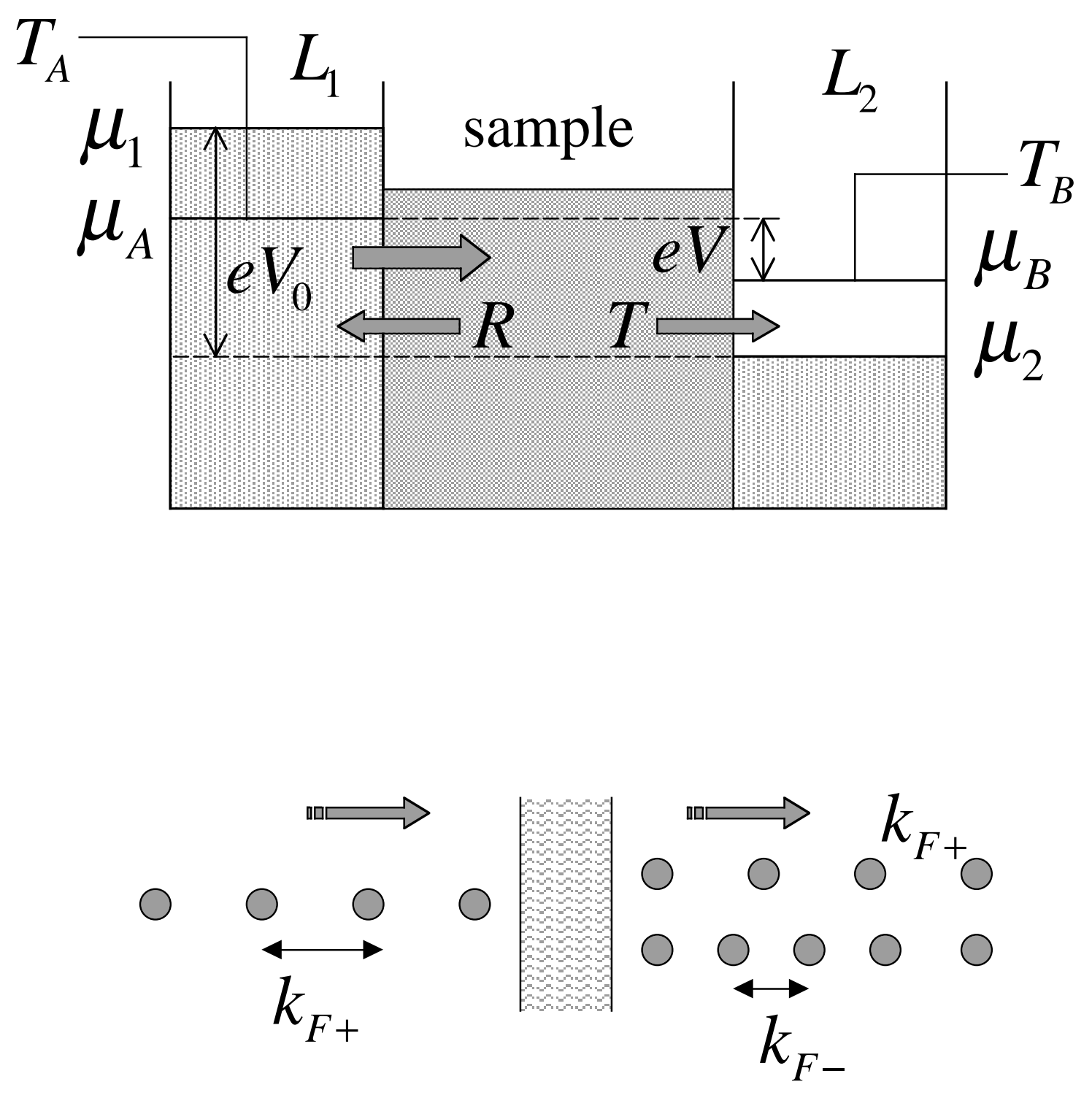




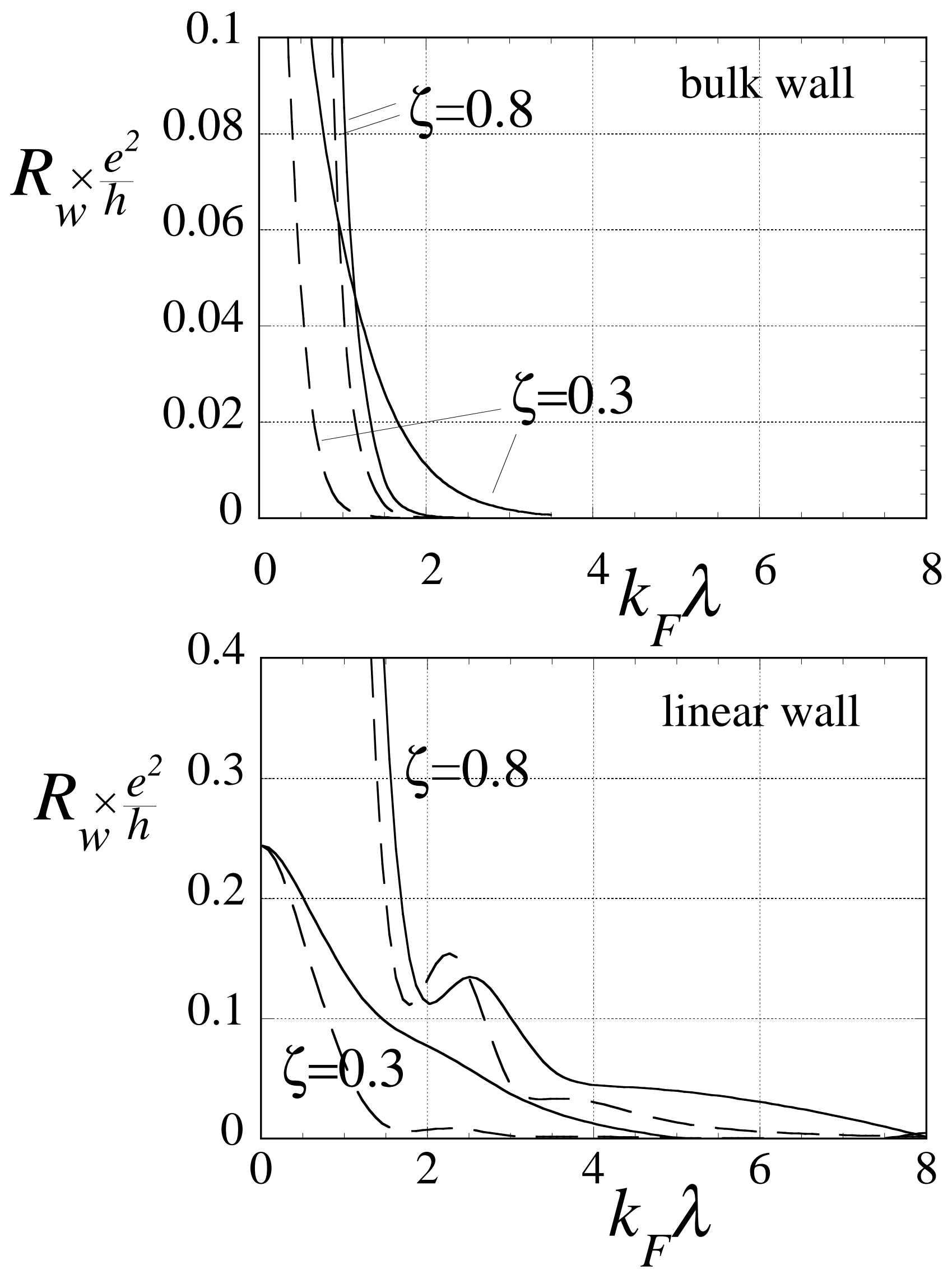

